Article

\title{
Interpretation of the Magnetic Field Signals Emitted by Encoded Asphalt Pavement Materials
}

\author{
Paulina Leiva-Padilla ${ }^{1, *} \mathbb{C}$, Fernando Moreno-Navarro ${ }^{1}$, Guillermo Iglesias ${ }^{2} \mathbb{D}$ and \\ $\mathrm{M}^{\mathrm{a}}$ Carmen Rubio-Gamez ${ }^{1}$ (D) \\ 1 Laboratory of Construction Engineering, University of Granada (LabIC.UGR), 18071 Granada, Spain; \\ fmoreno@ugr.es (F.M.-N.); mcrubio@ugr.es (M.C.R.-G.) \\ 2 Department of Applied Physics, University of Granada, 18071 Granada, Spain; iglesias@ugr.es \\ * Correspondence: pleiva@ugr.es
}

Received: 30 July 2020; Accepted: 4 September 2020; Published: 6 September 2020

\begin{abstract}
Asphalt materials modified with different types and dosages of magnetically responsive materials can emit patterns of magnetic signals easily detectable by magnetic field sensors. These patterns could be used to encode roads and improve infrastructure-to-vehicle (I2V)/road-to-vehicle (R2V) communications. In this sense, this paper presents a laboratory study addressed to analyze the magnetic field signals emitted by encoded asphalt specimens manufactured with various dosages of steel fibers. The analysis consisted in the evaluation of the influence of three parameters: (1) the height of placement of the magnetic field sensors, (2) the approach speed of the encoded specimen/vehicle and (3) the distance from signal detection. Results show that, for each one of the parameters evaluated, there is a limit value below which it is possible to work with the magnetic signal emitted by the encoded samples. A proof of concept was used to validate the results obtained.
\end{abstract}

Keywords: encoded asphalt materials; smart roads; road-to-vehicle; infrastructure-to-vehicle

\section{Introduction}

Five main benefits have been outlined from the implementation of autonomous vehicles (AV) [1]. The first benefit is related to the improvement of road safety [2,3] in terms of collision prevention between vehicles and infrastructure elements, pre-detection of vehicle malfunction and monitoring of the driver status during transit. The second benefit refers to easy driving and parking [4], which is associated with a reduction in fatigue, stress, and difficulties for the user. The third benefit is linked to operational efficiency $[5,6]$ as a result of reductions in fuel costs, insurance rates, and travel time. The fourth and fifth benefits are related to multitasking $[5,6]$ and social advantages for users $[2,3,5,6]$. For example, taking a break from driving, enjoying entertainment or an increase in work productivity, and reducing traffic congestion and fuel emissions, as well as improving the mobility of disabled or elderly people, and children.

The concerns identified with AVs relate to the vehicle's performance in bad weather conditions [4], safety issues linked to the vehicle's response to unexpected situations during driving [4,5,7] (i.e., the interaction with other self-driving vehicles and system failures), and privacy and security issues related to the occurrence of system failures [3,4] (i.e., errors or problems from hacking and violation of privacy). Additional concerns include the increase in the production and maintenance costs of the vehicles $[5,7]$ and the social challenges $[4,5,7]$ related to the legal liability associated with the ethical dilemma or social non-consensus produced by the use of these technologies. Furthermore, the definition of insurance policies and the difficulty of elderly or disabled people to use them also raise concerns.

In this sense, the work of the stakeholders within this industry is of upmost importance. Automotive manufactures and information technology companies in the United States and Europe 
have made considerable investments in the development of vehicle-to-everything communications (V2X) in the last 40 years $[1,8]$. The latest AV vehicle models are equipped with camera-based systems, geographic positioning systems (GPS), inertial navigation systems (INS), high-precision maps to identify obstacles, sensing technologies among them LIDAR, RADAR, ultrasonic and infrared sensors, as well as technologies already being implemented in conventional vehicles such as adaptative cruise control, lane assist, front collision warning, etc. $[2,9,10]$. This set of autonomous travel technologies are used to feed machine learning, big data, virtual reality, and other techniques to enhance the reliability of AVs [10-14].

Additionally, many government agencies and academia research centers in the United States and several European and Asian countries [1] have been working on infrastructure-to-vehicle (I2V)/road-to-vehicle (R2V) communications through investment in research, along with improvements in laws and regulations to promote the development of intelligent transportation infrastructure (ITS). Some of these systems are already used for the design and simulation of intelligent traffic at real intersections [15-17], as well as the implementation of Roadside Smart Devices (RSDs), which are composed of a large number of sensing devices in the road with short-range wireless communication for monitoring the physical phenomena happening in the road structure, as well as communicating with the vehicles [18-20]. Additional examples also include a hybrid between the visible light communication (VLC) and radio frequency (RF) systems [21-24], to offer longer distance transmissions and improved I2V.

In this respect, encoded asphalt materials are part of a recent solution for further assisting AV guidance, which consists of encoding part of the pavement structure by modifying asphalt mixtures with magnetically responsive materials $[9,25,26]$. This technique consists of producing certain magnetic field signals detectable by sensors installed in the vehicle. Previous studies [9] have already demonstrated that it is possible to codify asphalt materials using metallic particles (preferably with high iron content) and that the code established in this way is sufficiently sensitive to be read from regular magnetic field sensors. As a further step in the development of these encoded materials, the aim of this study is to analyze how pavement-vehicle interaction and vehicle speed influences the AV signal reception.

\section{Materials and Methods}

\subsection{Materials}

The capability of an encoded asphalt material to emit a magnetic field signal depends on the type of magnetically responsive material used in its manufacture. A previous study made by the authors [9] confirmed that steel fibers, thanks to their higher iron content, emit better magnetic field signals than steel slags. In this sense, the encoded materials selected for this study were manufactured with various dosages of steel fibers obtained from recycled end-of-life vehicle tires. The signals emitted by these encoded materials can be detected by sensors installed in vehicles and could act as a form of code to communicate the characteristics of a pavement (maximum speed, left or right turn in carriageway, type of road, etc.) (Figure 1). Therefore, based on this concept, there are unlimited codification possibilities to provide real-time information to the vehicles. 


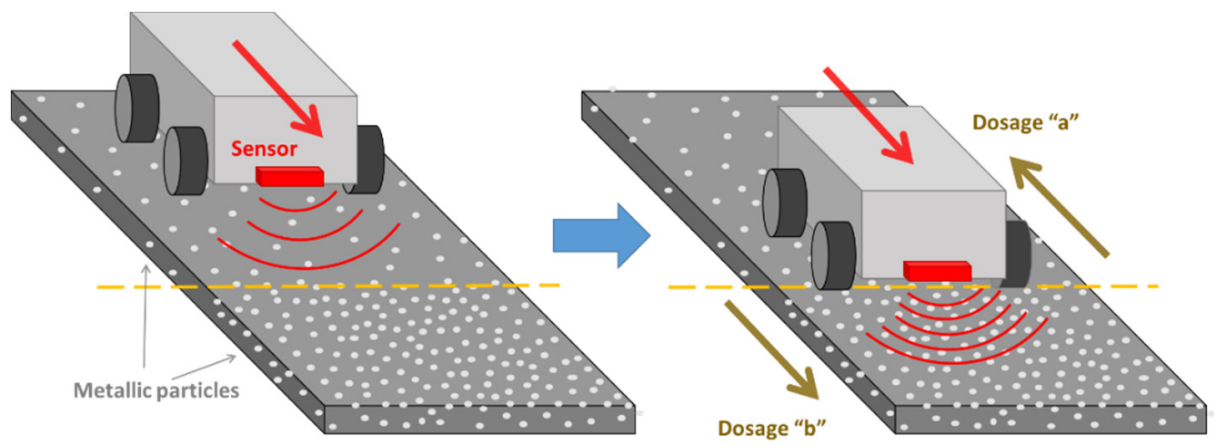

Figure 1. Characteristics of the pavements that can interact with sensors installed in the vehicles.

Figure 2 shows the steel fibers used, which were the smallest sizes obtainable from recycled tire plants. Their average lengths and diameters sizes were among 0.3 and $1.0 \mathrm{~cm}$, and 0.15 and $0.30 \mathrm{~cm}$, respectively. These fibers were used to manufacture mortar specimens of size $12 \times 8 \times 2 \mathrm{~cm}$, with percentages of $5 \%, 11 \%$ and $17 \%$ of steel fibers. The size of the steel fibers and the specimens used were selected to represent three main characteristics:

- Firstly, to create elements with a high concentration of steel fibers, which is required for magnetic field detection.

- Secondly, the fabrication of practical elements, which can be easily placed inside the surface course of existing pavements with minimal processing.

- Thirdly, the reduction in fiber grouping during mixing. This was achieved by preheating the fibers and incorporating them after the aggregates but before the asphalt binder in the mixing process. A discontinuous granulometry was used for the asphalt mixture, with fine aggregates (maximum size of $6 \mathrm{~mm})$ and a rich content of asphalt binder $(8 \%)$.

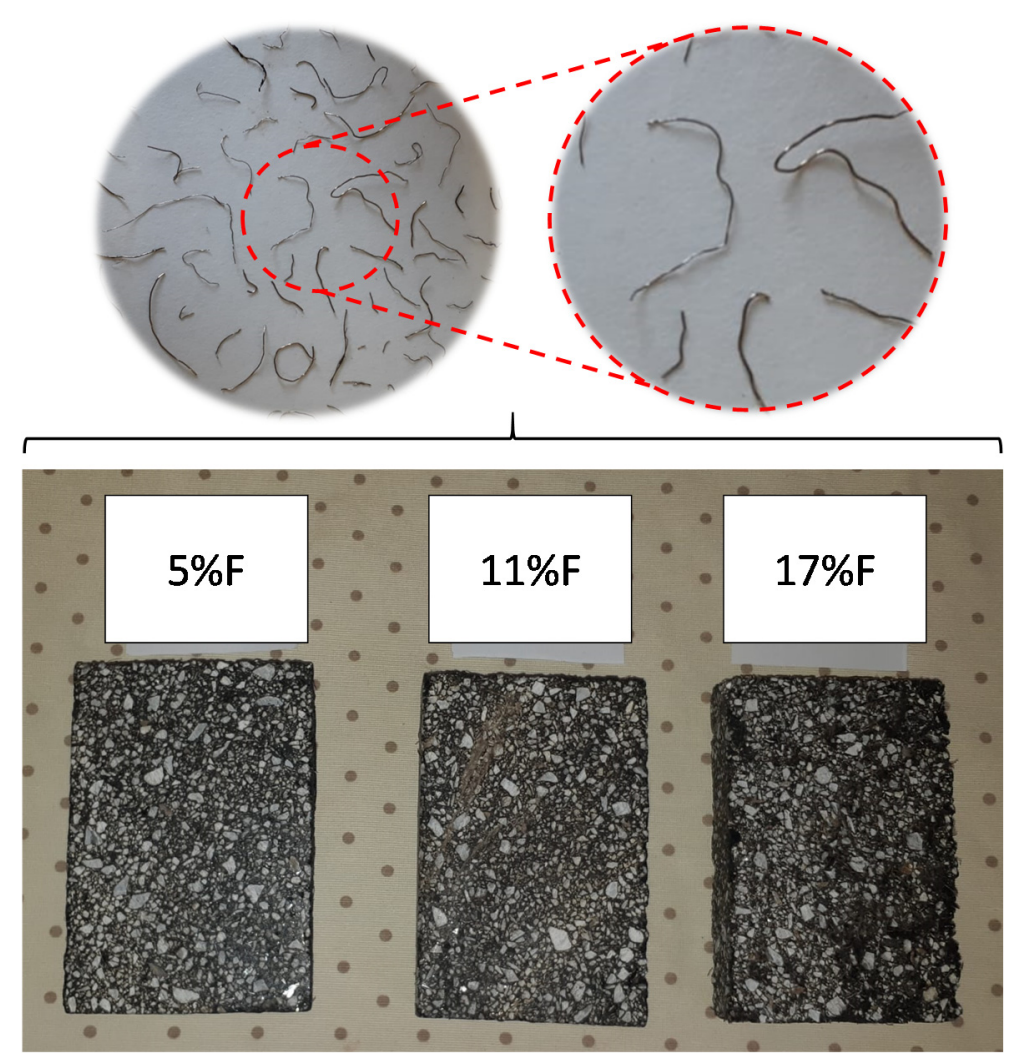

Figure 2. Steel fibers and the encoded asphalt samples used in the study. 
The asphalt binder used is a B50/70, with a penetration of $65 \mathrm{~mm}$ at $25^{\circ} \mathrm{C}$ based on the EN 1426 , a softening point of $51{ }^{\circ} \mathrm{C}$ based on the EN 1427, and a Fraass fragility of $-8{ }^{\circ} \mathrm{C}$ according to EN 12593 .

\subsection{Methods}

Given that the encoded asphalt materials are an emerging technology, which is still in the early stages of development, standardized test protocols to evaluate the magnetic signal emitted by these materials are not yet defined. In this sense, and also considering the conditions associated with the use of these materials inside the autonomous vehicle industry (for example, speed of approximation, the proper placement of the sensors, etc.), a novel test protocol was designed for this research study.

This test protocol required the design and manufacture of the system, as shown in Figure 3, which is composed of three main parts: (1) data measurement device, (2) data acquisition and emission device (via wireless) and (3) data capture and processing software.

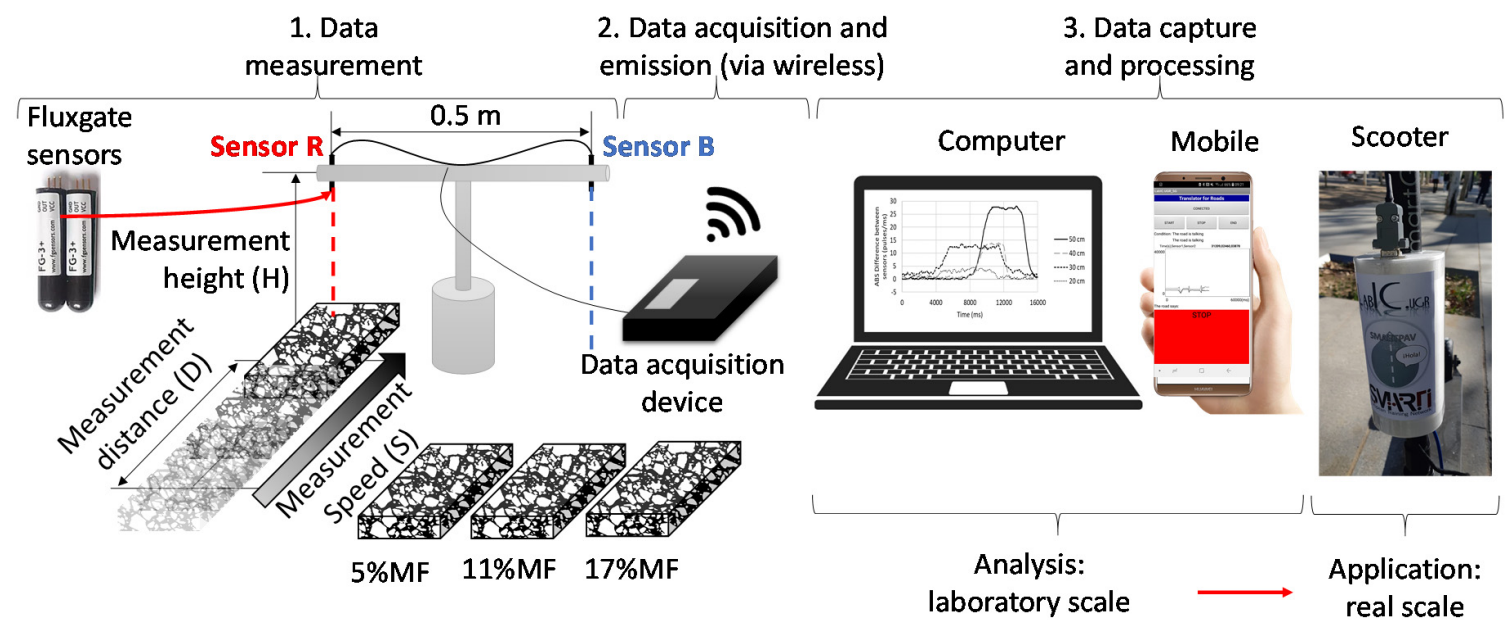

Figure 3. System manufactured for the study.

As shown in Figure 4, the first part consists of a base with two sensors separated at $0.5 \mathrm{~m}$ from each other to configure a horizontal gradiometer device. This horizontal gradiometer is capable of perceiving the vertical displacement of both sensors, where the field strength of the encoded specimen just in one of them. In this sense, it is possible to isolate the effect of the encoded specimen by scaling the measured values obtained from each sensor. The magnetic field sensors used are fluxgate sensors FG-3+, which operate in the range of \pm 50 microteslas. The output is a 5 -volt rectangular pulse with a period/frequency directly proportional to the field strength. This device was designed to measure the magnetic field signal emitted by the encoded samples according to three types of analysis shown in Figure 4:

- Analysis 1. Height influence: variation of the magnetic field strength depending on the height $(\mathrm{H}$ $=0.05 \mathrm{~m}, 0.1 \mathrm{~m}, 0.3 \mathrm{~m}$ and $0.4 \mathrm{~m}$ ).

- Analysis 2. Speed influence: variation of the magnetic field strength depending on the approach speed of the encoded sample $(\mathrm{S}=0.1 \mathrm{~m} / \mathrm{s}, 0.2 \mathrm{~m} / \mathrm{s}$ and $0.4 \mathrm{~m} / \mathrm{s})$.

- Analysis 3. Closer distance influence: variation of the magnetic field strength depending on the closer distance to the sensor (CD). As shown in Figure 4, the CD (dashed lines) depends on the measurement distance (D: Position $1=0.250 \mathrm{~m}$, Position $2=0.125 \mathrm{~m}$ and Position $3=0 \mathrm{~m}$ ) and the height of measurement $(\mathrm{H}=0.1 \mathrm{~m})$. 


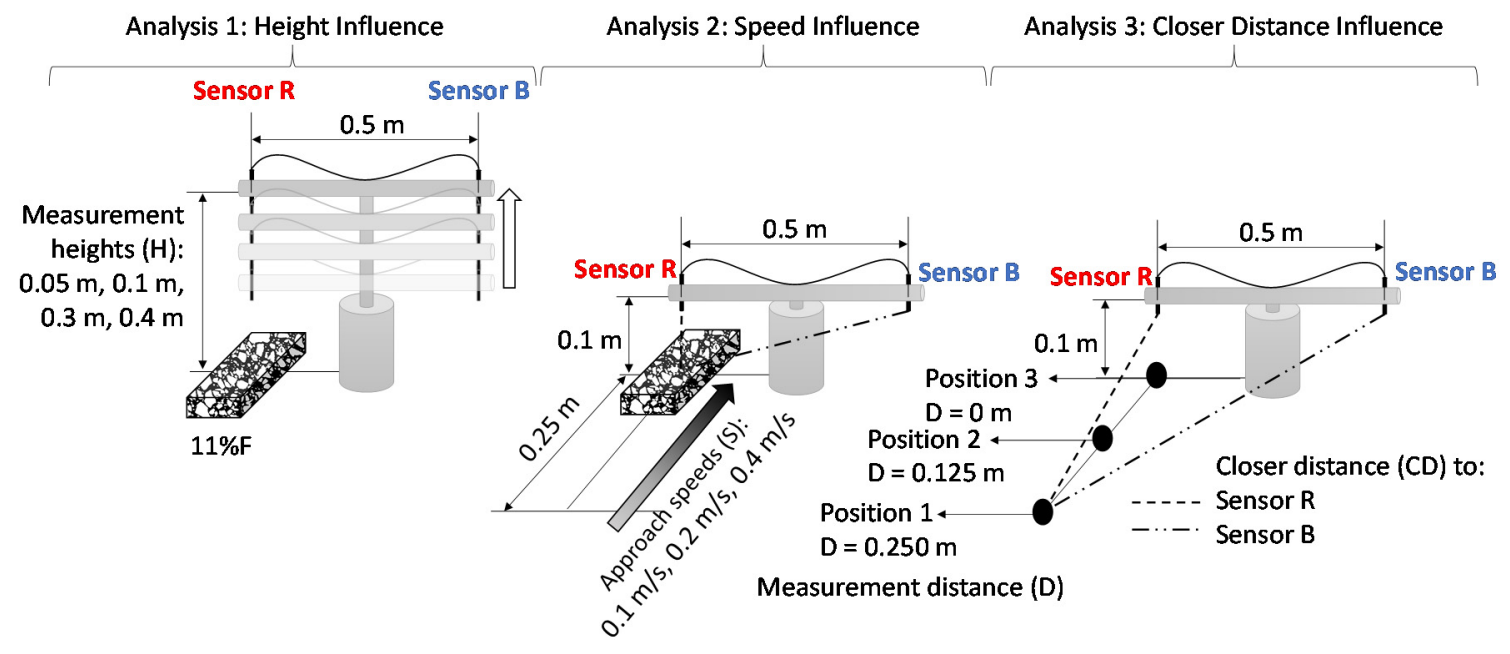

Figure 4. Process of analysis defined for this study.

The second part corresponds to the acquisition device responsible for the collection and emission of the data measured by the magnetic field sensors. As can be seen in Figure 5, this device was developed in Arduino, which is characterized to be an open access hardware and software platform, commonly used for the proposal of electronic prototypes addressed to multidisciplinary and multi-technology projects. Due to the range of frequency of the FG-3+ being higher than the range of commonly processed data by the Arduino UNO board, a PCB was designed (printed circuit board) in a shield format, which contains a frequency divisor and a Bluetooth module (Arduino HC06), to ensure the correct measurement of the data, as well as its wireless emission to the third part of the system.

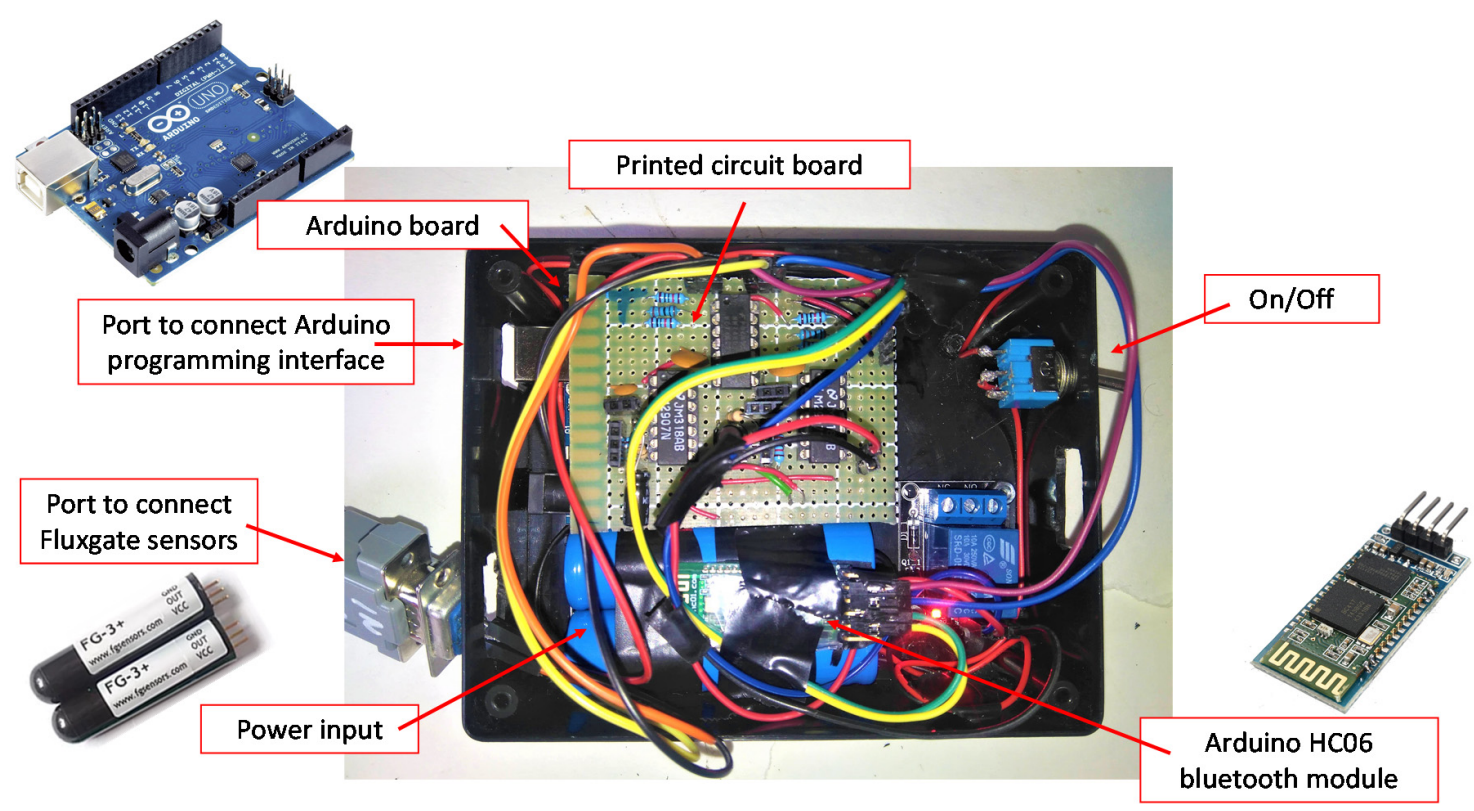

Figure 5. Details of the electronic components of the acquisition device.

The third part of the system involves the capturing and processing of the data, which will provide the final instructions that are proposed to help vehicle guidance. As shown in Figure 6a, the open source Arduino Software (IDE) was used to upload instructions to the Arduino UNO board and to extract the data required during the experiment. Additionally, Figure $6 \mathrm{~b}$ shows a mobile application (LabIC-SG) developed to capture and visualize data. This application was designed and programmed using the MIT App Inventor intuitive, visual programming environment developed by the Massachusetts Institute of Technology (MIT). 


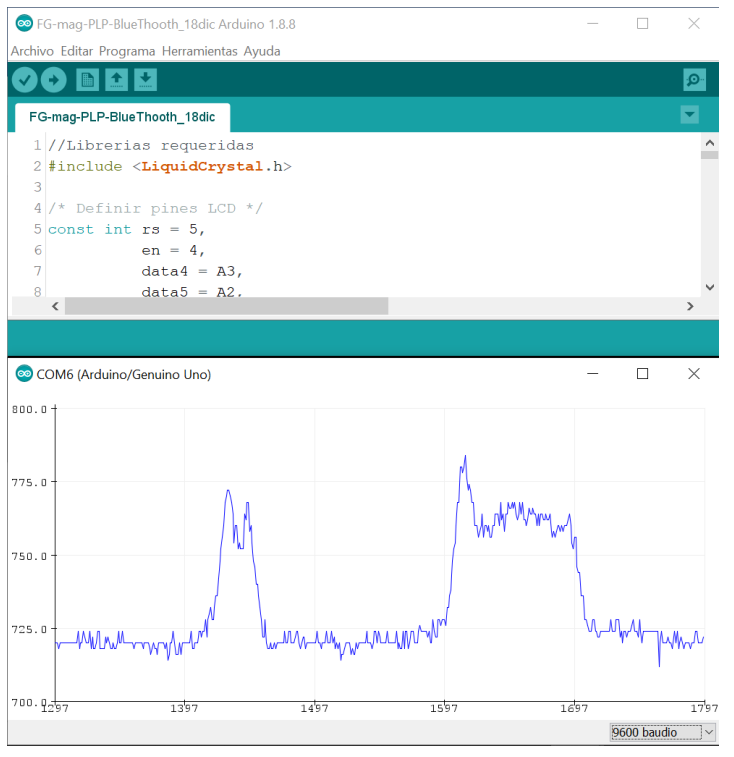

(a) Arduino IDE software

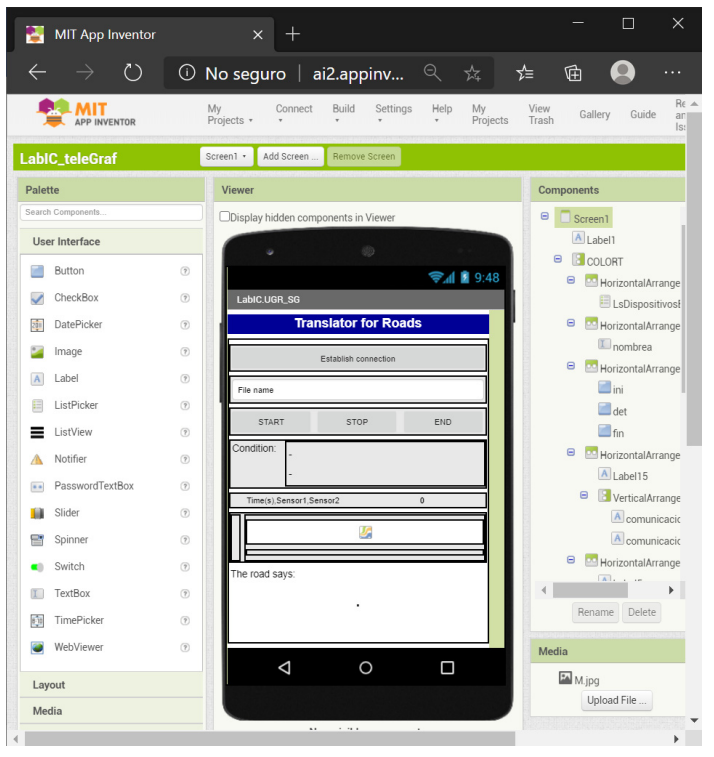

(b) MIT APPinventor platform

Figure 6. Software used and created for the data capture and processing

To test the functioning of the system, a 4-step proof of concept (PoC) was developed, as shown in Figure 7, for an electric scooter. Steps 1, 2 and 3 are related with the design, manufacture and assembly of the sensor installed in the electric scooter and the test track with the encoded plates. Step 4 is the final stage, which tests the correct functioning of the system.

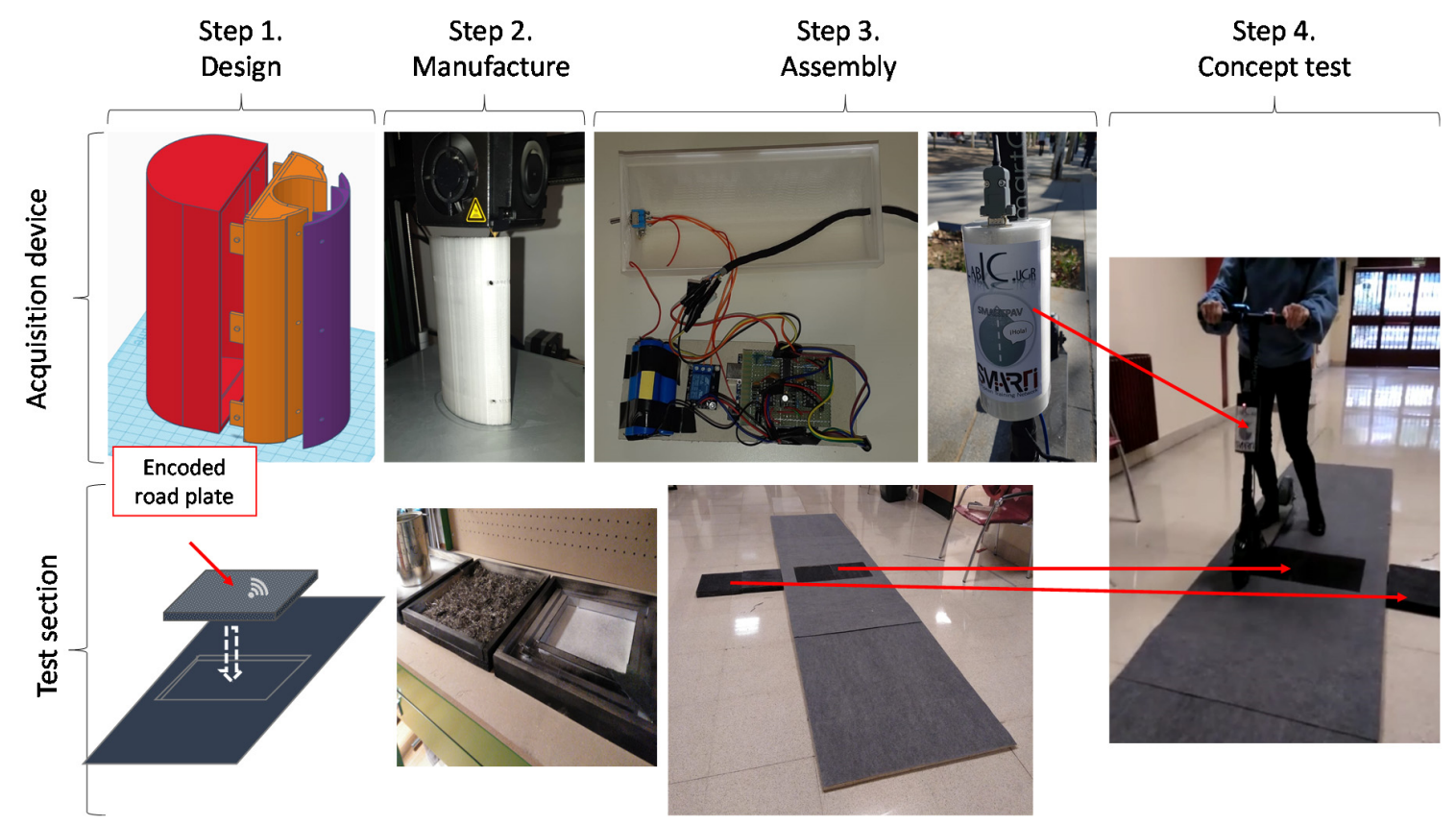

Figure 7. Four-step proof of concept.

\section{Results}

Figure 8 summarizes the maximum frequencies obtained for the specimen of $11 \% \mathrm{MF}$, when the measurement height $(\mathrm{H})$ of the sensor is increased. As can be observed, as $\mathrm{H}$ is increased, the frequency of detection $(\mathrm{kHz}$ : pulses $/ \mathrm{ms})$ in Sensor $\mathrm{R}$ is reduced, tending to a constant value of $718 \mathrm{kHz}$ at the highest $\mathrm{H}(0.4 \mathrm{~m})$ value, which means that the sensor does not recognize the specimen 
anymore. Therefore, the sensors should be positioned at measurement heights lower than approximately $0.11 \mathrm{~m}$

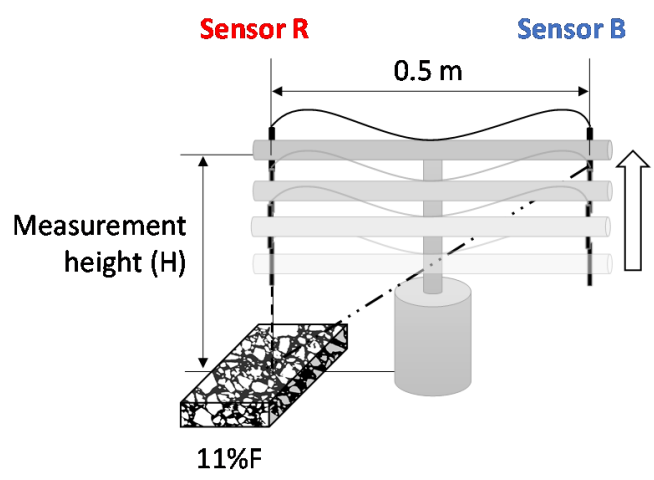

- Pulses for Sensor $R$ at $D=0 \mathrm{~m}$

....-.-Average frequency for Sensor B at D $=0 \mathrm{~m}$

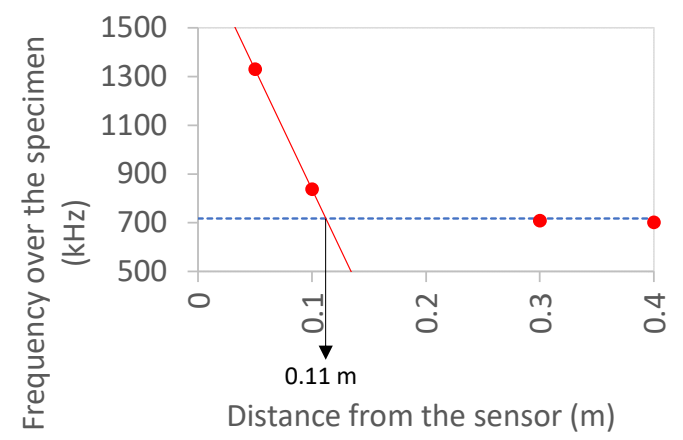

Figure 8. Influence of the measurement height $(\mathrm{H})$.

Figure 9 shows the recorded frequency values at varying approach speeds, with $\mathrm{H}=0.1 \mathrm{~m}$ and at a measurement distance of $0 \mathrm{~m}$. As can be seen, when the approach speed is increased, the recorded signal between both sensors is reduced. However, the frequencies could be increased by increasing the amount of magnetic material. It is important to remark that the values of the frequencies obtained are dependent on the electronic capacities of the sensors used.

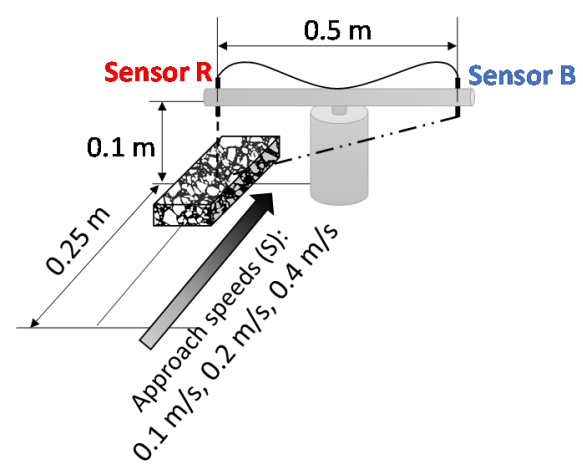

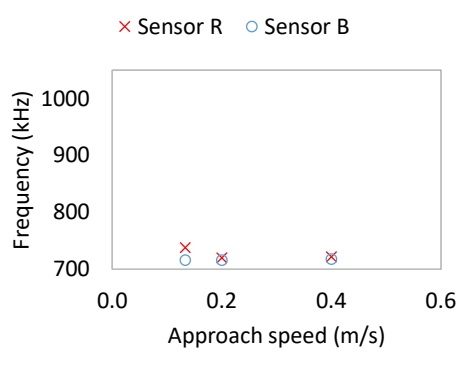

$5 \% \mathrm{~F}$

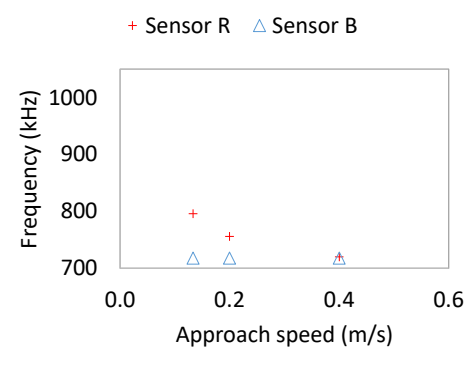

$11 \% \mathrm{~F}$

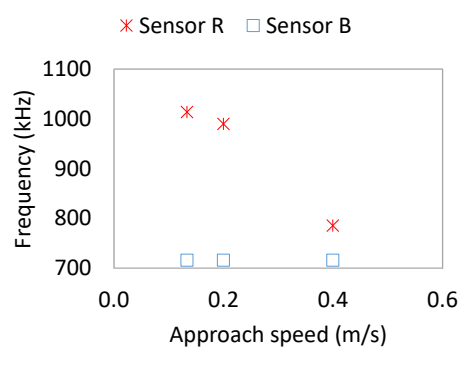

$17 \% \mathrm{~F}$

Figure 9. Influence of the measurement speed (S) at measurement distance $=0 \mathrm{~m}$.

Considering the same approach speeds evaluated in Figure 9, and to evaluate the capacity of localization of the device, the three specific positions for measuring frequency were selected, as shown in Figure 10. Results showed that, similar to the case of the variation of the measurement height (Figure 8), the closer the specimens are to the sensor (smaller CD), the higher the frequency values 
obtained. Once again, these values are dependent on the travel speed and the content of steel fibers used in the encoded specimens.

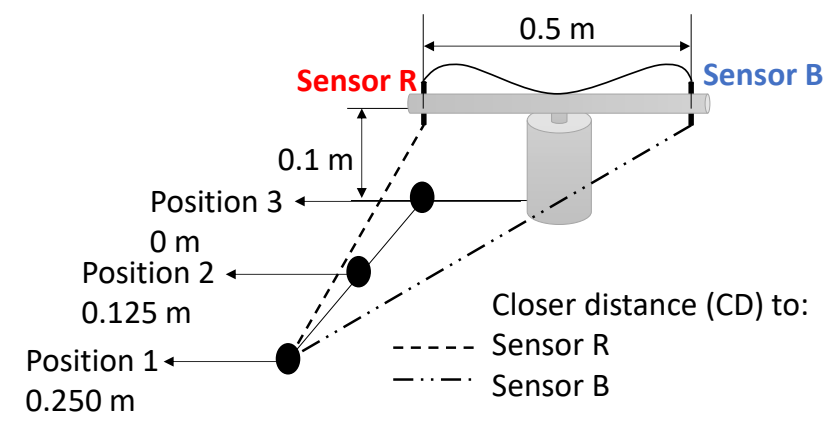

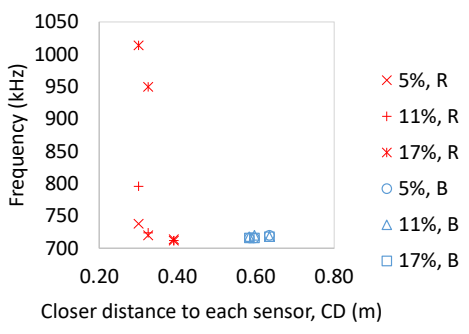

S: $0.1 \mathrm{~m} / \mathrm{s}$

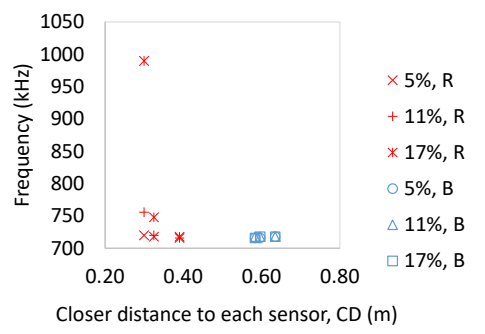

S: $0.2 \mathrm{~m} / \mathrm{s}$

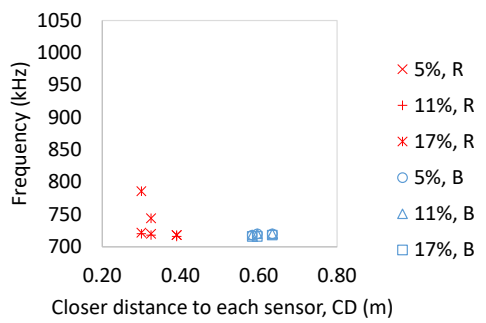

S: $0.4 \mathrm{~m} / \mathrm{s}$

Figure 10. Influence of the closer distance to each sensor.

Finally, an encoded plate with $11 \% \mathrm{~F}$ was manufactured and was placed in the test section to define a proof of concept with a binary instruction of activation/deactivation for an electric scooter. As can be seen in Figure 11, over the $740 \mathrm{kHz}(3.65 \mathrm{~V})$ the device installed in the scooter sends a high-voltage message to deactivate and stop the electric scooter.

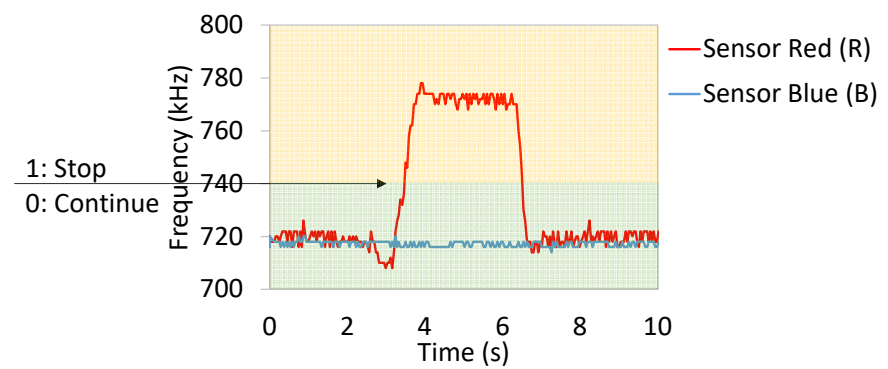

(a) Frequency data collected by the sensor

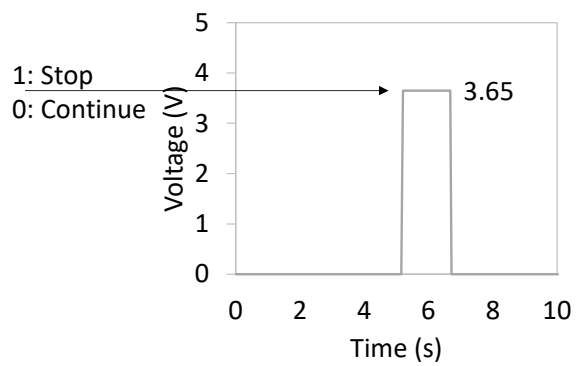

(b) Data treatment and conversion to voltage

Figure 11. Proof of concept based on binary activation for $11 \%$ F.

\section{Discussion}

In line with the aim of this study, the results presented have shown that there is an interaction between the encoded asphalt materials and the AV. This interaction is truly influenced by three variables studied, assessed with three different experiments, defined in this study's testing protocol.

In Analysis 1, it was demonstrated that there is a maximum limit related with the placement height of the magnetic field sensors. Over this limit value, the magnetic field sensors placed in the vehicle were not capable of detecting the magnetic field signal emitted by the encoded asphalt materials. Therefore, recognizing that the magnetic field signal of these materials depends on the type and dosage of the magnetically responsive material used in their manufacture [9], as well as the detection 
capacity of the magnetic field sensors [27-29], the limit of this variable could be readjusted according to the requirements of the system.

In Analysis 2 it was shown that, as the approach speed of the encoded specimen increases (which in real life would be the approach speed of the vehicle), the detection capacity of the magnetic field sensors is decreased. However, and aligned with the discussion of Analysis 1, it was confirmed that with a higher dosage of magnetically responsive material, the encoded asphalt materials' capacity to be detected by the magnetic field sensors was better.

In Analysis 3 it was determined that inside a distance of $0.4 \mathrm{~m}$ around the magnetic field, the signal of the encoded asphalt samples started to be detected. This means that there is an area of influence and time of detection before and after passing over the encoded area.

Finally, as a step towards the possible use of this technology in a real scale application, the end of the results section showed a successful implementation of a PoC developed with these materials. This achievement validates the use of encoded asphalt pavements for enhancing vehicle-infrastructure transit, which could have significant safety and operational benefits for AVs.

In this context, encoded asphalt materials could prove to be one of the required strategies to help increase the reliability of AV implementation. Road conditions, for example, traffic accidents, works on public roadways, environmental conditions, etc., are part of the challenges proposed to be addressed by Levels 3 and 4 in the scale of automation of the United Stated National Highway Traffic Safety Administration [30]. Therefore, keeping in mind that important investments and efforts that have been made by automotive manufacturers and information technology companies over the last few years, the development of more research related to the communication road-to-vehicle is still necessary to contribute to a real success in the implementation of AV. For example, and specifically related to encoded asphalt materials, further research could address the evaluation of the external noise in the signal, other materials that could potentially be used in the modification of the road, or alternative devices that can produce similar signals during their installation (i.e., electromagnets, plates with high density of magnetically responsive materials, etc.).

\section{Conclusions}

The present paper represents an additional step within a research project aiming to develop a new generation of encoded asphalt materials which could be used for the guidance of autonomous vehicles. Based on the results obtained, the following conclusions can be drawn:

- The magnetic field signal emitted by encoded asphalt materials depends on the placement height of the sensor, the vehicle approach speed and horizontal distance of the encoded asphalt specimens.

- A proof of concept validates the possible use of the encoded asphalt materials inside the required protocols to reach a more reliable implementation of AVs. This tool could be used in future work related to the development of this technology.

- Encoded asphalt materials, and their associated technologies, could greatly support road-to-vehicle communications and better help the successful implementation of AVs.

Author Contributions: All authors have contributed to the current paper. Conceptualization, P.L.-P., F.M.-N. and G.I.; Formal analysis, P.L.-P.; Funding acquisition, F.M.-N. and M.C.R.-G.; Investigation, P.L.-P., F.M.-N., G.I. and M.C.R.-G.; Methodology, P.L.-P., F.M.-N. and G.I.; Project administration, F.M.-N. and M.C.R.-G.; Resources, F.M.-N.; Supervision, F.M.-N. and G.I.; Writing—original draft, P.L.-P.; Writing一review \& editing, F.M.-N. and M.C.R.-G. All authors have read and agreed to the published version of the manuscript.

Funding: The research presented in this paper was carried out as part of the H2020-MSCA-ETN-2016. This project has received funding from the European Union's H2020 Programme for research, technological development, and demonstration under grant agreement number 721493.

Conflicts of Interest: The authors declare no conflict of interest. The funders had no role in the design of the study; in the collection, analyses, or interpretation of data; in the writing of the manuscript, or in the decision to publish the results. 


\section{References}

1. Kim, M.-K.; Park, J.-H.; Oh, J.; Lee, W.-S.; Chung, D. Identifying and prioritizing the benefits and concerns of connected and autonomous vehicles: A comparison of individual and expert perceptions. Res. Transp. Bus. Manag. 2019, 32, 100438. [CrossRef]

2. Bagloee, S.A.; Tavana, M.; Asadi, M.; Oliver, T. Autonomous vehicles: Challenges, opportunities, and future implications for transportation policies. J. Mod. Transp. 2016, 24, 284-303. [CrossRef]

3. Hohenberger, C.; Spörrle, M.; Welpe, I.M. Not fearless, but self-enhanced: The effects of anxiety on the willingness to use autonomous cars depend on individual levels of self-enhancement. Technol. Forecast. Soc. Chang. 2017, 116, 40-52. [CrossRef]

4. König, M.; Neumayr, L. Users' resistance towards radical innovations: The case of the self-driving car. Transp. Res. Part F Traffic Psychol. Behav. 2017, 44, 42-52. [CrossRef]

5. Howard, D.; Dai, D. Public Perceptions of Self-driving Cars: The Case of Berkeley, California. In Proceedings of the 93rd Annual Meeting of the Transportation Research Board Original Submission, Washington, DC, USA, 12-16 January 2014.

6. Bansal, P.; Kockelman, K.M.; Singh, A. Assessing public opinions of and interest in new vehicle technologies: An Austin perspective. Transp. Res. Part C Emerg. Technol. 2016, 67, 1-14. [CrossRef]

7. Ro, Y.; Ha, Y. A Factor Analysis of Consumer Expectations for Autonomous Cars. J. Comput. Inf. Syst. 2019, 59, 52-60. [CrossRef]

8. Van Brummelen, J.; O'Brien, M.; Gruyer, D.; Najjaran, H. Autonomous vehicle perception: The technology of today and tomorrow. Transp. Res. Part C Emerg. Technol. 2018, 89, 384-406. [CrossRef]

9. Moreno-Navarro, F.; Iglesias, G.R.; Rubio-Gámez, M.C. Encoded asphalt materials for the guidance of autonomous vehicles. Autom. Constr. 2019, 99, 109-113. [CrossRef]

10. Fényes, D.; Németh, B.; Gáspár, P.; Asszonyi, M. Possibilities of vehicle state estimation using big data approaches. In Proceedings of the 16th Mini Conference on Vehicle System Dynamics, Identification and Anomalies, Budapest, Hungary, 5-7 November 2018; pp. 395-401.

11. Arvind, C.S.; Senthilnath, J. Autonomous Vehicle for Obstacle Detection and Avoidance Using Reinforcement Learning. In Soft Computing for Problem Solving; Springer: Singapore, 2020; Volume 1048.

12. Guo, J.; Deng, W.; Zhang, S.; Qi, S.; Li, X.; Wang, C.; Wang, J. A Novel Method of Radar Modeling for Vehicle Intelligence. SAE Int. J. Passeng. Cars Electron. Electr. Syst. 2017, 10, 50-56. [CrossRef]

13. Lee, S.; Kim, Y.; Kahng, H.; Lee, S.K.; Chung, S.; Cheong, T.; Shin, K.; Park, J.; Kim, S.B. Intelligent traffic control for autonomous vehicle systems based on machine learning. Expert Syst. Appl. 2020, 144, 113074. [CrossRef]

14. Koopman, P.; Wagner, M. Autonomous Vehicle Safety: An Interdisciplinary Challenge. IEEE Intell. Transp. Syst. Mag. 2017, 9, 90-96. [CrossRef]

15. Grembek, O.; Kurzhanskiy, A.; Medury, A.; Varaiya, P.; Yu, M. Making intersections safer with I2V communication. Transp. Res. Part C Emerg. Technol. 2019, 102, 396-410. [CrossRef]

16. Bakibillah, A.S.M.; Kamal, M.A.S.; Tan, C.P.; Hayakawa, T.; Imura, J.-I. Event-Driven Stochastic Eco-Driving Strategy at Signalized Intersections from Self-Driving Data. IEEE Trans. Veh. Technol. 2019, 68, 8557-8569. [CrossRef]

17. Buzachis, A.; Celesti, A.; Galletta, A.; Fazio, M.; Fortino, G.; Villari, M. A multi-agent autonomous intersection management (MA-AIM) system for smart cities leveraging edge-of-things and Blockchain. Inf. Sci. (NY) 2020, 522, 148-163. [CrossRef]

18. Teng, H.; Liu, Y.; Liu, A.; Xiong, N.N.; Cai, Z.; Wang, T.; Liu, X. A novel code data dissemination scheme for Internet of Things through mobile vehicle of smart cities. Future Gener. Comput. Syst. 2019, 94, 351-367. [CrossRef]

19. Teng, H.; Liu, W.; Wang, T.; Liu, A.; Liu, X.; Zhang, S. A Cost-Efficient Greedy Code Dissemination Scheme through Vehicle to Sensing Devices (V2SD) Communication in Smart City. IEEE Access 2019, 7, 16675-16694. [CrossRef]

20. Teng, H.; Liu, W.; Wang, T.; Kui, X.; Zhang, S.; Xiong, N.N. A Collaborative Code Dissemination Schemes through Two-Way Vehicle to Everything (V2X) Communications for Urban Computing. IEEE Access 2019, 7, 145546-145566. [CrossRef] 
21. Momen, M.M.A.; Fayed, H.A.; Aly, M.H.; Ismail, N.E.; Mokhtar, A. An efficient hybrid visible light communication/radio frequency system for vehicular applications. Opt. Quantum Electron. 2019, 51, 364. [CrossRef]

22. Vieira, M.A.; Vieira, M.; Louro, P.; Vieira, P. Cooperative vehicular communication systems based on visible light communication. Opt. Eng. 2018, 57, 076101. [CrossRef]

23. Kim, Y.H.; Chung, Y.H.O. Experimental demonstration of highway I2V using visible light communications. Appl. Opt. 2016, 55, 5840-5845. [CrossRef]

24. Gheorghiu, R.A.; Bədescu, I.; Timnea, R.S. Infrastructure to vehicle communications using inductive loops. WSEAS Trans. Commun. 2014, 13, 596-605.

25. Leiva-Padilla, P.; Moreno-Navarro, F.; Iglesias, G.R.; Rubio-Gámez, M.C. Analysis of the mechanical response of asphalt materials manufactured with metallic fibres under the effect of magnetic fields. Smart Mater. Struct. 2019, 29, 015033. [CrossRef]

26. Leiva-Padilla, P.; Moreno-Navarro, F.; Iglesias, G. A Review of the Contribution of Mechanomutable Asphalt Materials towards Addressing the Upcoming Challenges of Asphalt Pavements. Infrastructures 2020, 5, 23. [CrossRef]

27. Li, J.; Wang, Y.; Zhang, X.; Ji, C.; Shi, J. Sensitivity and Resolution Enhancement of Coupled-Core Fluxgate Magnetometer by Negative Feedback. IEEE Trans. Instrum. Meas. 2019, 5, 23. [CrossRef]

28. Zhi, M.; Tang, L.; Qiao, D. Design and analysis of miniature tri-axial fluxgate magnetometer. Mod. Phys. Lett. B 2017, 31, 1750040. [CrossRef]

29. Rühmer, D.; Shanmuganathan, P.; Ludwig, F.; Schilling, M. Spatial and field resolution of wire-wound fluxgates in magnetic dipole fields. Sens. Actuators A Phys. 2012, 173, 30-35. [CrossRef]

30. NHTSA. Preliminary Statement of Policy Concerning Automated Vehicles; NHTSA: Washington, DC, USA, 2013.

(C) 2020 by the authors. Licensee MDPI, Basel, Switzerland. This article is an open access article distributed under the terms and conditions of the Creative Commons Attribution (CC BY) license (http://creativecommons.org/licenses/by/4.0/). 